\title{
Research on News Recommendation System Based on Deep Network and Personalized Needs
}

\author{
Weijia Zhang $\mathbb{D}^{1}$ and Feng Ling ${ }^{2}$ \\ ${ }^{1}$ City Institute, Dalian University of Technology, Dalian, Liaoning 116600, China \\ ${ }^{2}$ Dalian Daily, Dalian, Liaoning 116600, China \\ Correspondence should be addressed to Weijia Zhang; zwj1978@m.fafu.edu.cn
}

Received 2 September 2021; Revised 10 October 2021; Accepted 22 October 2021; Published 2 November 2021

Academic Editor: Yinghui Ye

Copyright (C) 2021 Weijia Zhang and Feng Ling. This is an open access article distributed under the Creative Commons Attribution License, which permits unrestricted use, distribution, and reproduction in any medium, provided the original work is properly cited.

\begin{abstract}
In order to solve the problems of poor performance of the recommendation system caused by not considering the needs of users in the process of news recommendation, a news recommendation system based on deep network and personalized needs is proposed. Firstly, it analyzes the news needs of users, which is the basis of designing the system. The functions of the system module mainly include the network function module, database module, user management module, and news recommendation module. Among them, the user management module uses the deep network to set the user news interest model, inputs the news data into the model, completes the personalized needs of the news, and realizes the design of the news recommendation system. The experimental results show that the proposed system has good effect and certain advantages.
\end{abstract}

\section{Introduction}

With the continuous development of the Internet, the mode or speed of information transmission has undergone earthshaking changes. Therefore, great changes have taken place in the way of obtaining information [1]. Coupled with the rapid development of mobile Internet and intelligent devices, people get information more quickly and can get news information anytime and anywhere. The value of news lies in real-time, so news communication is more valuable in the form of Internet development [2]. New media occupy a more and more important position in the modern media industry because of its rich forms, strong interaction, wide channels, high coverage, accurate arrival, high cost performance, and convenient promotion. The "new" of news media is reflected in the following three points: all-round digitization, interactivity, and personalization. The personalized information service provided by this news media enables the audience to obtain personalized customized services. The news media based on the Internet not only brings convenience for users to obtain information but also brings the problem of information overload, and this problem is more serious than the traditional media. In the face of a large amount of news information, users cannot quickly find the information they need. The existing Mengguang website provides the same news information to different users and also provides a certain retrieval function, which meets the needs of users to a certain extent, but still cannot meet the deep-seated needs of users [3]. Under a large amount of news information, how to quickly classify and mine massive information and then push it to different users has become an urgent need to be solved. A recommendation system is a system that mainly solves the problem of information overload on the Internet. It can filter the information on the Internet, obtain more user interest preferences, and push the information similar to the user's interest to the user, so as to effectively control the accumulation of ineffective information in the user's field of vision [4]. Recommendation system is divided into a nonpersonalized recommendation system and a personalized recommendation system. The nonpersonalized recommendation system provides recommendations with the same content to all users. The personalized recommendation system can recommend thousands of people and thousands of faces according to users [5]. At 
the same time, users can be grouped through user clustering, and people with the same hobbies can be placed in the same group. Because the effect of personalized recommendation is very good, it has been widely used in existing Internet products. Therefore, in order to improve the efficiency of news recommendation, a lot of research has been done on news recommendation system in this field [6].

Literature [7] proposed a personalized news recommendation based on event ontology. Aiming at the problems of cold start, sparse data, lack of semantics, and low recommendation accuracy in the traditional recommendation system, a recommendation algorithm based on event ontology is proposed. The event ontology is constructed by combining the news classification structure and news corpus, the elements of the news browsed by users are extracted, and the user interest model is constructed. The classification structure based on event ontology calculates the similarity between news events. The user interest similarity is calculated through the user interest model, and the relevant news events are found according to the semantic radius of the nonhierarchical structure of the event ontology. The news personalized recommendation results are obtained from the three aspects of event ontology similarity, user interest similarity, and nonhierarchical structure similarity. The experimental results show that the recommendation results of this algorithm are better than the collaborative filtering recommendation algorithm and content-based recommendation algorithm. This recommendation method does not consider the personalized needs of users, and there are more uninterested information in the recommended news information. Literature [8] proposed a hybrid recommendation algorithm based on incremental collaborative filtering and latent semantic analysis. The hybrid recommendation algorithm dynamically adjusts the recommendation list by incrementally updating the item similarity list in the item-based incremental update collaborative filtering module. The experimental results show that the proposed IULSACF algorithm is better than the traditional recommendation method in various evaluation indexes. This method takes less consideration of news recommendation and has some limitations. Literature [9] proposed the design and implementation of the personalized news recommendation system based on collaborative filtering. The key research contents include proposing a personalized news recommendation model, improving traditional collaborative filtering algorithm, and realizing a personalized news recommendation system combined with a mobile platform. The personalized news recommendation model includes four modules, namely, news classification, user interest analysis, user clustering, and recommendation result generation. The improvement of collaborative filtering algorithm includes filling the default value with the average user score and the popularity of items to solve the problem of data sparsity and setting the time weight of user score data with the time attenuation function to solve the problem of user interest migration. The implementation of a personalized news recommendation system includes analyzing the requirements of the system, introducing the design ideas and implementation methods of the system, integrating the improved recommendation algorithm into the system, and finally realizing the development of a personalized news recommendation system. This paper proposes a personalized news recommendation model and improves the traditional collaborative filtering algorithm. Finally, a personalized news recommendation system based on collaborative filtering is designed and implemented on the basis of the Android system. The personalized news recommendation system designed and implemented in this paper is in line with the era background of mobile Internet, can bring users a personalized service experience, and has high research value and application significance. Literature [10] made an in-depth study of the literature from 2001 to 2019 and listed 81 relevant studies, which were roughly divided into six categories for discussion and solved some of the many challenges in the field of journalism. Literature [11] proposed a new personalized news recommendation framework, the Hybrid Personalised News Recommendation (HYPNER). In Literature [12], the author proposes a context-mixing, deep-learning-based method for sessionbased news recommendation, which can utilize multiple information types.

The Internet of Things (IoT) is an information carrier based on the Internet, traditional telecommunication networks, etc. It enables all ordinary physical objects that can be independently addressed to form interconnected networks. The application of the Internet of Things involves all aspects. The application of the Internet of Things in the fields of industry, agriculture, environment, transportation, logistics, security, and other infrastructure effectively promotes the intelligent development of these aspects, makes the limited resources more rationally used and allocated, and thus improves the efficiency and benefits of the industry. In the household, medical and health care, education, finance and service industry, tourism and other fields closely related to life, service scope, service mode, and service quality have been greatly improved, greatly improving people's quality of life. With the progress of science and technology in human society, the development of the Internet of Things has become an irresistible trend.

In order to solve the shortcomings of the above recommendation, this paper designs a news recommendation system based on deep network and personalized needs. Firstly, it analyzes the news needs of users, which is the basis of designing the system. The functions of the system module mainly include network function module, database module, user management module, and news recommendation module. Among them, the user management module uses the deep network to set the user news interest model, inputs the news data into the model, completes the personalized needs of the news, and realizes the design of the news recommendation system. The experimental results show that the proposed system has a good effect and certain advantages.

Our contribution includes the following three points:

(1) In order to solve the problems of poor performance of the recommendation system caused by not considering the needs of users in the process of news recommendation, a news recommendation system 
based on the deep network and personalized needs is proposed

(2) We use the deep network to set up the user's news interest model, input the news data into the model, complete the personalized needs of news, and realize the design of the news recommendation system

(3) The experimental results show that the proposed system has good effect and certain advantages

\section{Research on News Recommendation System Based on Depth Network and Personalized Needs}

2.1. Demand Analysis of the News Recommendation System. The content application of news shows all the news in the platform to users according to category and time. Users can retrieve the content according to their favorite news categories and the navigation displayed by the news system and search directly for news with specific keywords or clear content. However, the problem brought by this news system is that all users see the same news information list. It does not process news information for different users and allows the news system to customize their own news homepage for each user $[13,14]$. The personalized news recommendation system will obtain the user's list of news of interest according to the user's demographic information and usage records on the platform. In this paper, the collaborative filtering algorithm is used for personalized news content recommendation. It recommends relevant news content to users according to the correlation between users or the correlation between news. The advantage of this method is that it can quickly build the similarity between users and recommend the news information viewed by similar users to each other.

The main requirements of the personalized news recommendation system are as follows:

(1) The system can recommend real-time news for users according to their current browsing behavior, so as to ensure that users can quickly obtain real-time news

(2) The news recommendation list can be updated in time, and the user recommendation list can be calculated according to the way that the news system collects Xinli, so as to ensure that users can obtain the latest recommendation list after logging in the Xinyang system every time

(3) The system should better solve the problem of news recommendation for new users and the problem of user cold start

(4) The newly added news also needs to be quickly recommended to users to solve the long tail effect in news information and better solve the cold start problem of the project

(5) The system tracks the accuracy and recall rate of the recommendation system [15], ensures that the news

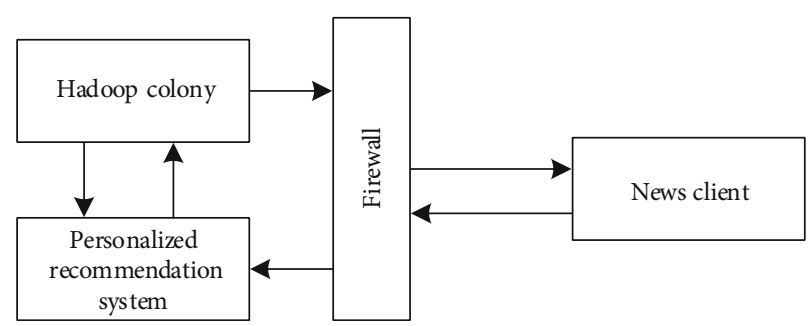

FIGURE 1: Network function design of the news recommendation system.

recommendation has a high accuracy and recall rate, and makes users satisfied with the current recommendation

(6) The system should be able to adapt to a large number of users and new news on the platform and can quickly calculate the push, save, and proofread type

(7) The system has good expansibility and meets the system's ability to deal with SeaView data in terms of capacity and computing power

(8) The real-time computing $\mathrm{H}$-frame is used to calculate the personalized recommendation model, so as to improve the actual push and storage capacity of the whole platform

2.2. Function Module Design of News Recommendation System. In order to realize the effectiveness of the news recommendation system, based on the existing hardware, the system designs a network function module, database module, user management module, and news recommendation module.

2.2.1. Network Function Module of the News Recommendation System. The network is a key module of its implementation, and the fast and slow response of the network response reflects the performance of the system. Therefore, this system network takes advantage of the computing power and storage power of the Hadoop cluster [16] and stores the final computing results in a NoSQL database with a high concurrency power like HBase. The personalized news recommendation system modifies the relevant task parameters and submits the task to the Hadoop platform for computation, while the platform needs to monitor whether the entire recommended calculation is working properly. The Hadoop platform is responsible for the calculation and storage of the recommendation models and regularly conducts the recommendation model calculation to ensure the normal operation of the recommendation model calculation and finally stores the calculation results in [17] in HBase. The news client of the recommendation system is displayed by pulling the relevant user news recommendation list from HBase. The personalized network deployment diagram is shown in Figure 1.

2.2.2. News Recommendation System Database Module. The database in the news recommendation system is its recommended core function module. Therefore, the recommended 


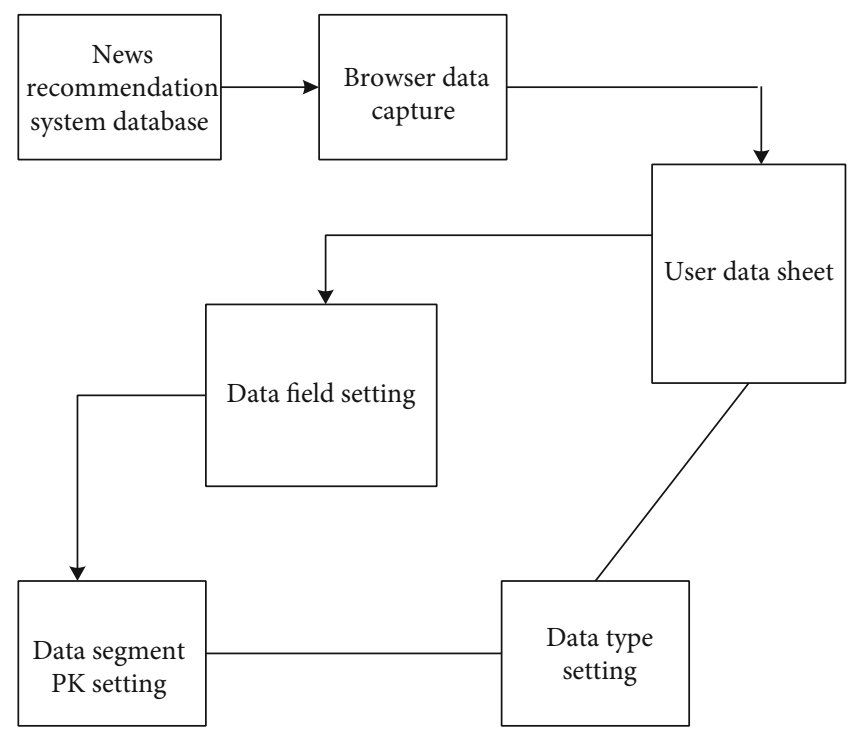

Figure 2: Recommended system database structure.

TABle 1: User data.

\begin{tabular}{lccc}
\hline $\begin{array}{l}\text { The field } \\
\text { name is called }\end{array}$ & Data type & $\begin{array}{c}\text { Do you have the } \\
\text { main key }\end{array}$ & $\begin{array}{c}\text { Field } \\
\text { description }\end{array}$ \\
\hline ID & INT & $\mathrm{Y}$ & User number \\
Account & VARCHAR11 & $\mathrm{N}$ & Login ID \\
Password & VARCHAR32 & $\mathrm{N}$ & Login password \\
Salt & VARCHAR20 & $\mathrm{N}$ & Password salt \\
Name & DATETIME & $\mathrm{Y}$ & Name of user \\
Type & TINYTIME & $\mathrm{N}$ & Role type \\
Create time & DATETIME & $\mathrm{Y}$ & Creation time \\
\hline
\end{tabular}

system provides a detailed design of the database module. All data in this system is stored as [18] via MySQL, with hot data and user data successfully logged in in Redis, for easy user access. The database structure of the system is shown in Figure 2.

The news recommendation system database mainly includes user data, permission data, and news information data; user data and news data are shown in Tables 1 and 2.

\subsubsection{User Management Module Based on a Deep Network.} In the user management module of the recommendation system, the personalized needs of users are mainly managed, and their interests are analyzed, so as to be the recommended object of the recommendation system. In user management, the news word vector is trained first, needing to divide the news text and remove the stop words included therein first. This paper uses the three deactivated word tables of HIT, the Baidu deactivated word table, and the machine intelligence laboratory of Sichuan University, combined to obtain a comprehensive deactivated word table. We place words in news text into the deword table to match. After removing the stop words, word vectors were trained using the Word2Vec model in Gensim. In order not to miss keywords, the minimum word frequency of the model was set to 1 , the number of iterations to 5 , and the word vector dimension to 200, and the model was trained to obtain the word vector model [19], and the calculated word vectors can be described as

$$
V=\left[v_{1}, v_{2}, \cdots v_{200}\right]
$$

Every user will have a certain interest tendency; manifested in the news system is the click and reading tendency of the news content. How to measure users' interest is the key to recommendation algorithms. This article treats the news content that the user has viewed as inner ci of interest, generating three vectors of interest for each user through records of the user browsing, i.e.,

$$
I=\frac{\sum_{I}^{N} W_{i}}{N},
$$

where $I$ represents the number of set elements of all words included in the user's reading record, $N$ represents the set of word vectors of all words included in the user's reading record, and $W_{i}$ represents word vectors.

Based on this, this paper manages and trains user data with the help of a deep neural network. The structure of the deep network is composed of a number of restricted Boltzmann machines, in which the network layer can be divided into two layers: visual layers, and follows the rules of no connection and connection between layers. Usually, the hidden layer unit is mainly used to train the higherorder correlation characteristics shown by extracting visual layer data, as shown in Figure 3.

The average vector was calculated as the user vector of interest based on the deep network structure determined above. We enter the user vector of interest data into a deep network, convert the one-dimensional user vector into an 
TABLE 2: News data.

\begin{tabular}{|c|c|c|c|}
\hline $\begin{array}{l}\text { The field } \\
\text { name is } \\
\text { called }\end{array}$ & Data type & $\begin{array}{l}\text { Do you have } \\
\text { the main key }\end{array}$ & $\begin{array}{c}\text { Field } \\
\text { description }\end{array}$ \\
\hline ID & INT & $\mathrm{Y}$ & News number \\
\hline Title & VARCHAR45 & $\mathrm{N}$ & Head(ing) to come \\
\hline Content & VARCHAR32 & $\mathrm{N}$ & News content \\
\hline Author & VARCHAR20 & $\mathrm{N}$ & Author name \\
\hline Image-url & DATETIME & $\mathrm{Y}$ & Figure connection \\
\hline Browse & TINYTIME & $\mathrm{N}$ & Browse number \\
\hline $\begin{array}{l}\text { Create } \\
\text { time }\end{array}$ & DATETIME & $\mathrm{Y}$ & Creation time \\
\hline
\end{tabular}

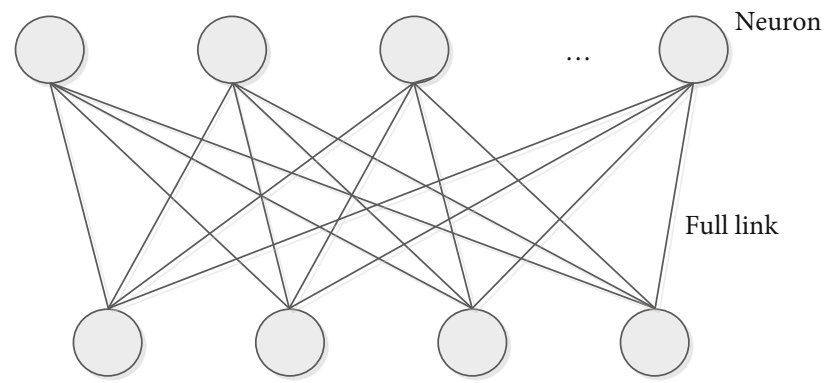

Figure 3: Basic structure of the deep network.

ordinary dimensional vector, and obtain it by using the convolutional operation in the deep network:

$$
C_{i}=f\left(w \times X_{i, j, h-1}+b\right) .
$$

Among these, $C_{i}$ represents the eigenvalues in the eigenmap, $w$ represents the deep network kernel function, and $b$ represents the sliding window.

At this time, the acquired feature vector is

$$
C=\left[c_{1}, c_{2}, \cdots c_{n-h-1}\right] .
$$

According to the determined user interest feature vector, the user news interest determination model is constructed to obtain

$$
C_{P}=\left[c_{1}, c_{2}, \cdots c_{n-h-1 / 2}\right]
$$

It is then trained through a deep network, yielding the following:

$$
L=-\sum_{1}^{N} Y_{i} \log \left(y_{i}\right)
$$

Among them, $N$ represents the number of news and $y_{i}$ represents the degree of user love.

2.2.4. News Personalized Recommendation Module. According to the above analysis, the subject model was established and trained, the new input news is subject classified, and the content of the news is preprocessed; then, through the

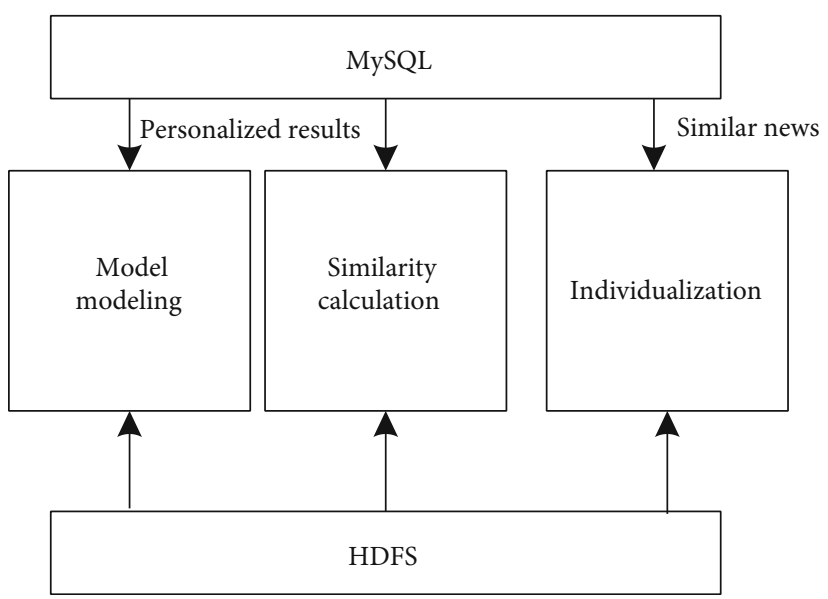

FIgURE 4: User-personalized recommended module structure.

subject model classification, the feature vectors of the news are stored into the file system HDFS. The personalized recommended news list is completed, filtering by collaborative filtering methods based on two aspects of the user and item. Finally, the obtained news list is filtered and integrated, the processed structure is newly ranked, and the news list to the user personalized recommendation module is shown in Figure 4 .

\section{Experimental Analysis}

3.1. Experimental Scheme. To test the validity of the proposed system, an experimental analysis was performed. The hardware and software environment for the experimental test are shown in Table 3.

The network architecture of the experimental test system is shown in Figure 5.

3.2. Analysis of Experimental Results. In order to highlight the effectiveness of the method, it is compared against the accuracy of the system in [7] and the system in [8], and the results are shown in Figure 6.

The experimental results in Figure 6 show that with the continuous change of analysis time, the accuracy of user demand data recommendation using this system against the literature [7] system and the literature [8] system is different in news recommendation. Among them, the accuracy of the system of user demand data in news recommendation is about $98 \%$. The best accuracy of the user demand data recommendation in the news recommendation is at least about $89 \%$. The accuracy of user demand data recommendation is about $80 \%$. By contrast, the accuracy of the system in the news recommendation is $9 \%$ higher than the literature [7] system and $18 \%$ above the literature [8] system. In contrast, the system recommended better results. This is due to the user management module using a deep network setting user news interest model in this system design. The news data is entered into the model, the personalized needs for news are completed, the design of the news recommendation system is realized, and the effectiveness of the system is improved. 
TABLE 3: Test environment settings.

Hardware settings

Software settings

Database

The amount of news data (GB)
Dell, Intel i7, internal storage, internal memory, EMS memory: 8 GB CentOS 6.4, MySQL

SQL

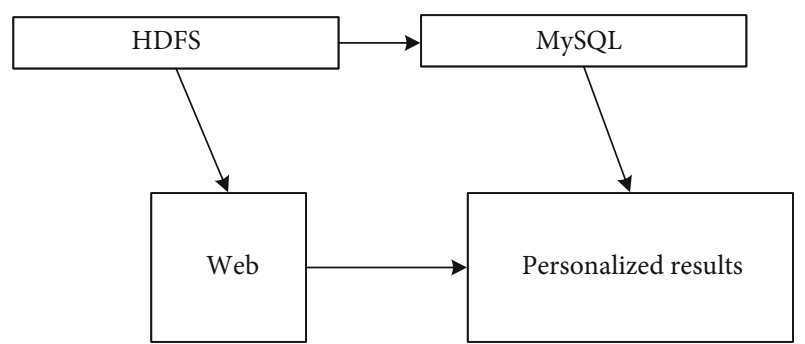

Figure 5: Test system environment.

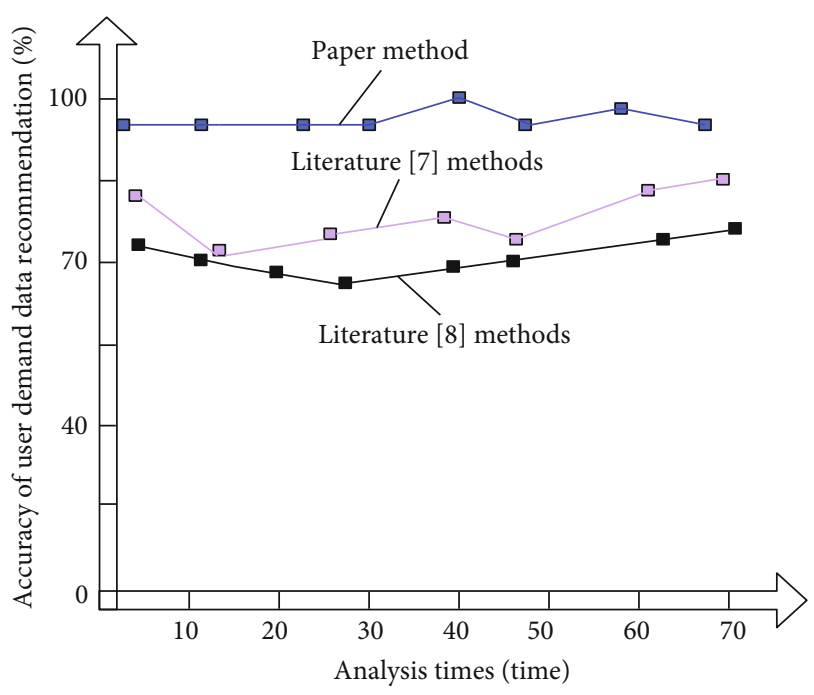

FIgURE 6: Accuracy analysis of user demand data recommendations in news recommendations.

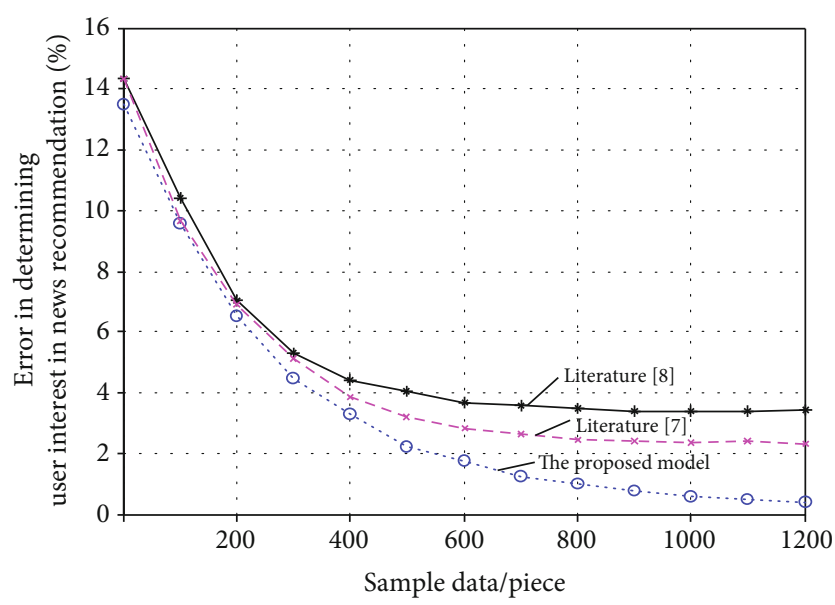

FIGURE 7: Error analysis of user interest determination in the news recommendations.
The experiment compares the errors of user interest in this system, literature [7] system, and literature [8] system in news recommendation. The results are shown in Figure 7.

The experimental results in Figure 7 show that the errors determined in the user interest of the paper system, literature [7] system, and literature [8] system are different. When the sample data size is constantly changing, the error is determined by user interest in news recommendations. Among them, the error of user interest in the news recommendation is about $0.1 \%, 2.1 \%$ in the literature [7] system, and about $3.9 \%$ in the news recommendation. This is the result of the analysis in the system design and verifies the effectiveness of the system.

\section{Conclusion}

In order to solve the problem of a deep network and personalized demand, resulting in the poor performance of the news recommendation system, a news recommendation system is proposed. First, we analyze the user news needs as the basis of the system design; the system module functions mainly include the network function module, database module, user management module, and news recommendation module. Among them, the user management module uses the deep network to set up the user news interest model, inputs the news data into the model, completes the personalized needs of the news, and realizes the design of the news recommendation system. The experimental results show that the proposed system is highly effective and has certain advantages. Although our method has achieved good experimental results, with the development of deep learning today, we should integrate the deep learning method into the algorithm of this paper. In the future, we will devote ourselves to this endeavor.

\section{Data Availability}

The data used to support the findings of this study are available from the corresponding author upon request.

\section{Conflicts of Interest}

The authors declare that they have no conflict of interest.

\section{References}

[1] K. Han, "Personalized news recommendation and simulation based on improved collaborative filtering algorithm," Complexity, vol. 2020, Article ID 8834908, 2020.

[2] H.-S. Sheu, Z. Chu, D. Qi, and S. Li, "Knowledge-guided article embedding refinement for session-based news 
recommendation," IEEE Transactions on Neural Networks and Learning Systems, vol. 99, pp. 1-7, 2021.

[3] J. Fergie, A. Howard, L. Huang, and A. Srivastava, "Implementation experience with meningococcal serogroup B vaccines in the United States: impact of a nonroutine recommendation," The Pediatric Infectious Disease Journal, vol. 40, no. 3, pp. 269-275, 2021.

[4] C. Song, W. Liu, Z. Liu, and X. Liu, "User abnormal behavior recommendation via multilayer network," PLoS One, vol. 14, no. 12, article e0224684, 2019.

[5] A. Gcs and B. Ml, "Stacked DeBERT: all attention in incomplete data for text classification," Neural Networks, vol. 136, no. 1, pp. 87-96, 2021.

[6] M. A. Ibrahim, M. U. Ghani Khan, F. Mehmood, M. N. Asim, and W. Mahmood, "GHS-NET a generic hybridized shallow neural network for multi-label biomedical text classification," Journal of Biomedical Informatics, vol. 116, no. 1, p. 103699, 2021.

[7] Z. H. U. Wenyue, L. I. U. Wei, and L. I. U. Zongtian, "News personalized recommendation based on event ontology," Computer Engineering, vol. 45, no. 6, pp. 267-272, 2019.

[8] L. I. U. Hui, W. A. N. Cheng-feng, and W. U. Xiao-hao, "A hybrid recommendation model based on incremental collaborative filtering and latent semantic analysis," Computer Engineering and Science, vol. 41, no. 11, pp. 2033-2039, 2019.

[9] L. I. U. Jin-hui, C. U. I. Xiang-yang, Y. A. N. G. Fan, and L. I. U. Li-yan, "Design and implementation of a combined recommendation system based on spring boot and user portrait," Electronic Component and Information Technology, vol. 3, no. 5, pp. 24-29, 2019.

[10] C. Feng, M. Khan, A. U. Rahman, and A. Ahmad, "News recommendation systems-accomplishments, challenges \& future directions," IEEE Access, vol. 8, pp. 16702-16725, 2020.

[11] A. Darvishy, H. Ibrahim, F. Sidi, and A. Mustapha, "HYPNER: a hybrid approach for personalized news recommendation," IEEE Access, vol. 8, pp. 46877-46894, 2020.

[12] G. D. S. P. Moreira, D. Jannach, and A. M. Da Cunha, "Contextual hybrid session-based news recommendation with recurrent neural networks," IEEE Access, vol. 7, pp. 169185-169203, 2019.

[13] N. J. Krishna, H. Purohit, and H. Rangwala, "Diversity-based generalization for neural unsupervised text classification under domain shift," vol. 15, no. 24, pp. 1145-1152, 2020, http://arxiv.org/abs/2002.10937.

[14] I. Chalkidis, M. Fergadiotis, S. Kotitsas, P. Malakasiotis, N. Aletras, and I. Androutsopoulos, "An empirical study on large-scale multi-label text classification including few and zero-shot labels," vol. 45, no. 2, pp. 123-128, 2020, http://arxiv.org/abs/2010.01653.

[15] I. Baldini, D. Wei, K. N. Ramamurthy, M. Yurochkin, and M. Singh, "Your fairness may vary: group fairness of pretrained language models in toxic text classification," vol. 45, no. 1, pp. 15-21, 2021, http://arxiv.org/abs/2108.01250.

[16] R. Asgarnezhad, M. Soltanaghaei, and S. A. Monadjemi, "An application of MOGW optimization for feature selection in text classification," The Journal of Supercomputing, vol. 16, no. 15, pp. 154-160, 2020.

[17] M. Oleynik, A. Kugic, Z. Kasáč, and M. Kreuzthaler, "Evaluating shallow and deep learning strategies for the $2018 \mathrm{n} 2 \mathrm{c} 2$ shared task on clinical text classification," Journal of the American Medical Informatics Association, vol. 26, no. 11, pp. 12471254, 2019.
[18] T. Igamberdiev and I. Habernal, "Privacy-preserving graph convolutional networks for text classification," vol. 7, no. 1, pp. 868-871, 2021, http://arxiv.org/abs/2102.09604.

[19] Q. I. U. Ning-jia, G. A. O. Peng, W. A. N. G. Peng, and T. A. O. Yue, "Research on ACO-WNB classification algorithm based on improved information gain," Computer Simulation, vol. 36, no. 1, pp. 295-299, 2019. 